\title{
Direct puncture for diagnosis of intradural spinal arachnoid cyst and fenestration using 3D rotational fluoroscopy: technical note and the "jellyfish sign"
}

\author{
Ziev B. Moses, MD, John H. Chi, MD, MPH, and Ram V. S. R. Chavali, MD \\ Department of Neurosurgery, Brigham and Women's Hospital, Harvard Medical School, Boston, Massachusetts
}

The authors report on a 47-year-old woman with a symptomatic thoracic spinal arachnoid cyst (SAC) who underwent a novel procedure that involves direct puncture of the SAC to visualize, diagnose, and potentially treat these rare spinal lesions. The method described utilizes 3D fluoroscopy to gain access to the SAC, followed by injection of myelographic contrast into the cyst. A characteristic "jellyfish sign" was observed that represents the containment of the contrast within the superior aspect of the cyst and a clear block of cranial flow of contrast, resulting in an undulating pattern of movement of contrast within the cyst. Following balloon fenestration of the cyst, unimpeded flow of contrast was visualized cranially throughout the thoracic subarachnoid space. The patient was discharged the following day in good condition, and subsequently experienced 1 year free from symptoms. This is the first reported case of a successful direct puncture of an SAC with balloon fenestration, and the first noted real-time fluoroscopic "behavior" of CSF within an arachnoid cyst. https://thejns.org/doi/abs/10.3171/2018.4.SPINE171182

KEYWORDS spine; arachnoid cyst; intradural; percutaneous; minimally invasive; direct puncture; surgical technique

T NTRADURAL spinal arachnoid cysts (SACs) are infrequent lesions typically located in the dorsal thoracic spine that often present with symptoms of spinal compression. ${ }^{15}$ If untreated, these lesions can result in permanent spinal cord damage. While imaging studies including MRI and CT myelography may suggest the diagnosis, diagnostic difficulties arise when the cyst wall or communicating pedicle is not well visualized on MRI. ${ }^{10} \mathrm{CT}$ myelography can be helpful in the diagnosis but often requires delayed imaging after contrast injection to delineate the cyst. ${ }^{13}$ Furthermore, differentiating SACs from idiopathic spinal cord herniation (ISCH) can be difficult using traditional methods, each of which requires a different treatment approach. ${ }^{1,6}$ For SAC removal, an inability to distinguish the cyst wall from normal subarachnoid space prior to surgery precludes one from more narrowly tailoring the surgical approach to only the involved levels and minimizing the size of the laminectomy.

For these challenging cases, we have begun offering a minimally invasive percutaneous procedure to diagnose these lesions, and in the case of SAC, we demonstrate a potential concurrent treatment. Performed in our inter- ventional neuroradiology suite, we take advantage of 3D rotational fluoroscopic guidance to puncture the SAC, inject intracystic contrast under real-time fluoroscopy, and confirm restoration or a change of flow postintervention. Two other case reports of percutaneous aspiration and/or fenestration of SACs have been published; however, these failed to describe real-time dynamic behavior of these cysts and confirmatory postprocedure restoration of flow between the cyst and subarachnoid space. ${ }^{4,14}$ We visualize the dynamic pulsatile ("jellyfish") nature of an SAC on real-time imaging and for the first time report a balloon dilation technique for SAC fenestration with evidence of postfenestration communication between the remnant cyst and subarachnoid space.

\section{Case Presentation}

\section{History and Examination}

This 47-year-old woman experienced an approximately 5-year history of slowly progressive lower extremity weakness. Over the years what initially had troubled her as a gait disturbance progressed to right-sided foot drop and 

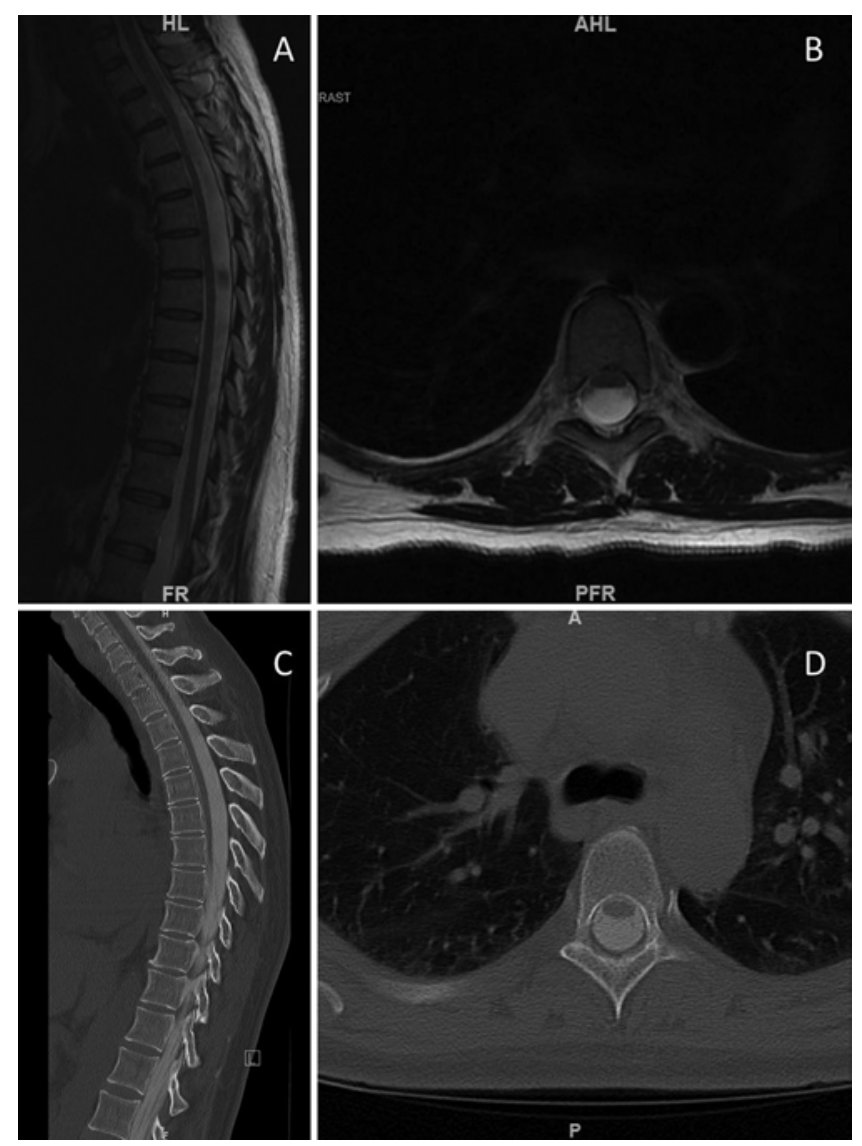

FIG. 1. A: Sagittal T2-weighted MR image displaying expanded subarachnoid space and anterior displacement of the spinal cord. B: Axial T2-weighted MR image at the level of T5. C: Sagittal CT myelogram displaying contrast opacification throughout the subarachnoid space. D: Axial CT myelogram.

then bilateral lower extremity weakness. This was initially attributed to amyotrophic lateral sclerosis given a positive family history, but after further neurological workup and negative testing, a thoracic spine MR image was obtained that suggested a compressive extramedullary cystic lesion
(Fig. 1A and B) centered at T5. At presentation, she was no longer as active and had stopped participating in sports. On examination, she was found to have brisk lower extremity reflexes, right foot drop, a T5 sensory level, and endorsed urinary incontinence.

A CT myelogram was obtained and revealed anterior cord flattening from T2 through T6 with opacification of the entire subarachnoid space and inability to rule out ISCH (Fig. 1C and D). Given ongoing treatment of breast cancer with chemotherapy and her reluctance to undergo open surgery, she proceeded to undergo two percutaneous cystic aspirations using biplane fluoroscopy with 3D guidance. After initial improvement in strength and gait for approximately 3 weeks, the patient called to report that her symptoms had returned to baseline. This response occurred on a second aspiration as well, and after repeat MRI demonstrated a similar appearance of the dorsal cyst, we were prompted to proceed with a percutaneous cystic fenestration.

\section{Technical Details}

With the patient prone on our 3D biplane fluoroscopy table (General Electric), we located the T5 spinous process and identified the target T4-5 interspace. Because of the limited 3D field of view, metallic BBs were used to mark the site (Fig. 2A). Next, using proprietary planning software (General Electric; Fig. 2B), a 20-gauge spinal needle was advanced under biplane fluoroscopy into the intradural space at T4-5 with spontaneous flow of clear fluid.

Next, myelographic contrast was injected, clearly demonstrating the cranial boundary of the cyst wall (Fig. 3A) and a unique and characteristic undulating movement for which the senior author coined the term "jellyfish sign." There is clear containment of the contrast within this superior aspect of the cyst and a clear block of cranial flow of contrast (see Video 1 for anteroposterior [AP] and lateral views).

VIDEO 1. Clip showing four different parts of the procedure: 1) AP view of contrast filling the superior aspect of the cyst with evidence of myelographic block; 2) lateral view of contrast filling the superior aspect of the cyst with evidence of myelographic block; 3) a 3-mm Sterling microballoon dilation catheter being passed over the previ-
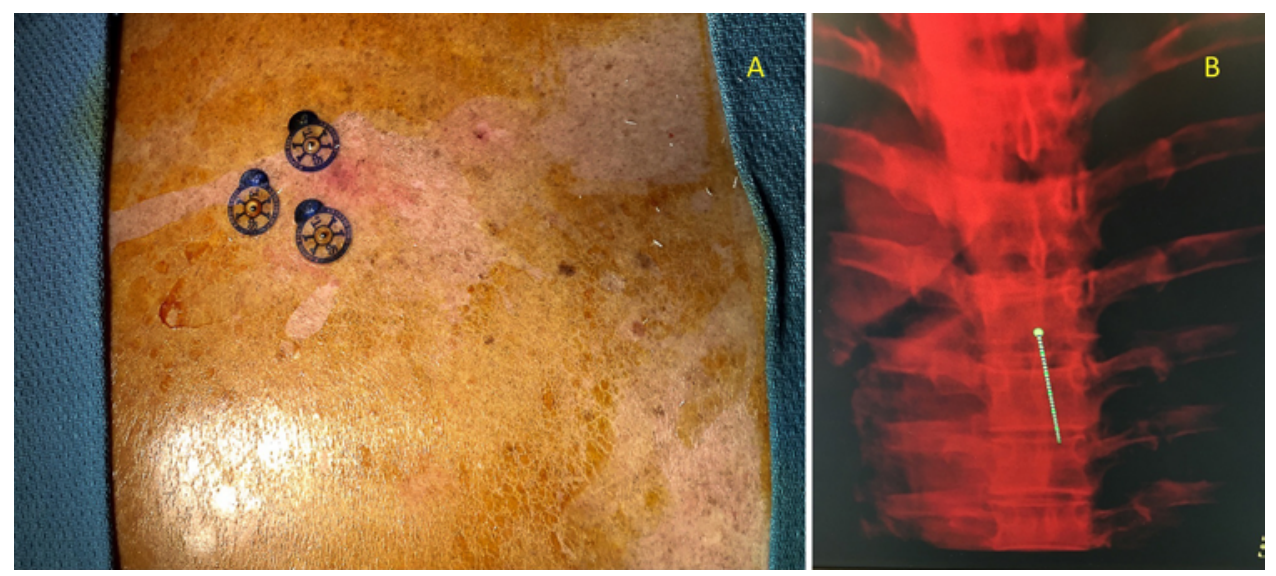

FIG. 2. A: Photograph of radiopaque markers on the patient's skin demarcating the T4-5 interspace. B: Planning trajectory (yellow line) from the skin to the intradural space. Figure is available in color online only. 

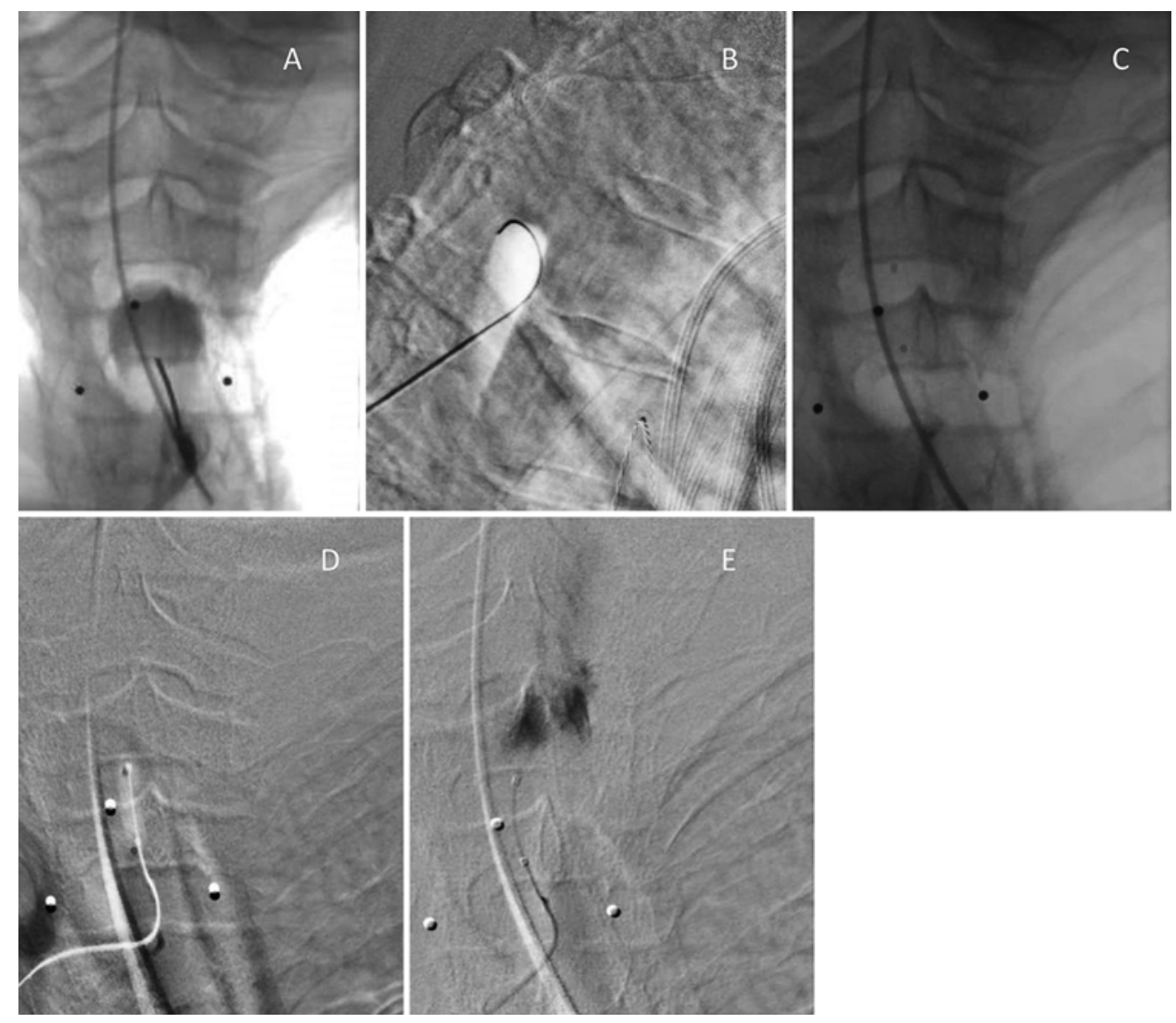

FIG. 3. A: AP fluoroscopic view of contrast filling the cranial aspect of the cyst with myelographic block. B: Lateral view of the threaded flexible wire within the cyst with the option of leaving the wire as a localizer for surgery. C: AP view of the balloon catheter (demarcated by two radiopaque intraluminal markers) through the cranial portion of the cyst wall. D: AP view of the inflated balloon catheter. E: AP view shows free flow through the cranial aspect of the cyst into the subarachnoid space after fenestration.

ously passed flexible wire to the cranial aspect of the cyst under digital subtraction imaging in preparation for balloon fenestration; and 4) myelographic contrast injected after fenestration revealing free flow of contrast medium cranially throughout the thoracic subarachnoid space. Copyright Ram V. S. R. Chavali. Published with permission. Click here to view.

Subsequently, the 0.018-inch guidewire provided with a standard vascular micropuncture kit (Cook Medical) was advanced into the cyst (Fig. 3B), and after tract dilation we used a standard Seldinger technique to advance a monorail 3-mm Sterling microballoon dilation catheter (Video 1) to the cranial aspect of the cyst under subtraction imaging. With the aid of the stiff mandrel supplied with the balloon catheter, the cyst wall was punctured and finally the microballoon was advanced across the cyst into the normal "free" subarachnoid space. After confirming flow of clear CSF spontaneously through the side port (Fig. 4), the balloon was inflated to create a $3-\mathrm{mm}$ fenestration through the cyst wall (Fig. 3C and D). The catheter was then withdrawn into the cyst cavity and contrast was injected, revealing unimpeded flow of contrast cranially throughout the thoracic subarachnoid space (Fig. 3E, Video 1).

\section{Follow-Up}

The patient was admitted overnight and uneventfully discharged the next day in stable physical condition, with unchanged right foot drop. At the 6-month follow-up evaluation, the patient reported continued improvement with less foot drop. However, at the 1-year follow-up she is reporting recurrence of symptoms and MRI suggests recurrence of her cyst.

\section{Discussion}

The preferred treatment strategy for intradural SACs is open laminectomy over the involved vertebral levels and attempted total removal, including the cyst wall. In cases

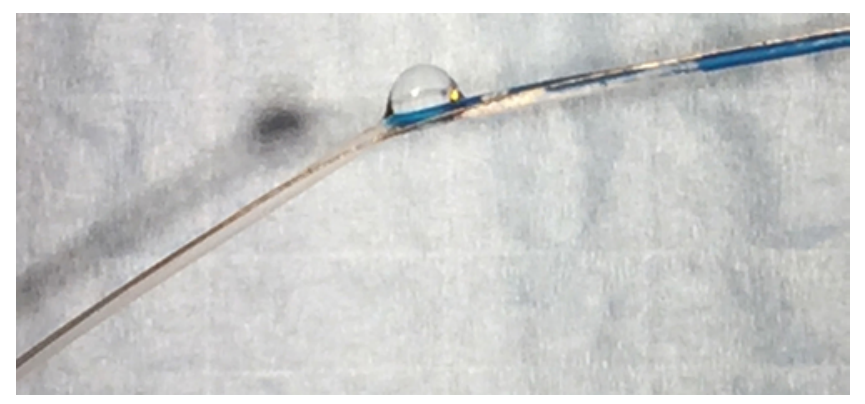

FIG. 4. Image of clear CSF droplet collecting at the balloon catheter side port following fenestration. Figure is available in color online only. 
of adherent dorsal arachnoid cysts, dorsal cysts spanning many levels in which a laminectomy may result in instability (e.g., in one study the average cranial-caudal extension was 3.7 levels $^{3}$ ), or ventral cysts that may be difficult to approach, wide fenestrations are favored over the risks associated with total removal. ${ }^{11}$ However, the role of percutaneous aspiration and/or fenestration remains unclear in SAC treatment. Some authors propose that for small cysts without obvious subarachnoid communication, aspiration alone may be feasible, $, 4,14$ whereas others have argued that these cysts are more likely to have a secretory epithelium, arguing against aspiration as a sole treatment modality.,11

Two prior reports of SAC aspiration under MRI guidance have been described. ${ }^{4,14}$ In both instances, the arachnoid cysts were well defined, without obvious communication to the subarachnoid space, and spanned a short segment. Bellavia et al. ${ }^{4}$ aspirated a cyst in the dorsal thoracic spine with intradural and extradural components, whereas Takahashi et al. ${ }^{14}$ both aspirated and performed a simple fenestration using a spinal needle in the anterior cervical spine where open surgery would incur higher risk. In both cases, dynamic imaging of the cyst was not obtained and confirmation of treatment success was judged by diminution of the cyst on intraprocedural MRI. Follow-up imaging at 9 months and 1 year showed no recurrence of the cyst.

In comparison, our SAC spanned a longer cranio-caudal dimension of 4 vertebral levels, communicated vigorously with the subarachnoid space without clear delineation of the cyst boundaries, and was resistant to two prior simple aspirations. Our approach is the first described use of 3D fluoroscopy to aid in both the diagnosis and treatment of SAC. Three-dimensional guidance allowed for planning an accurate trajectory to the intradural space. Direct cystomyelography allowed real-time confirmation and diagnosis of the cyst and following fenestration, dynamic imaging confirmed cyst disruption and the loss of the functional myelographic block with free cranial flow of contrast medium.

For the first time we directly visualize the dynamic nature of the cyst as it opacifies and undulates in a manner resembling a jellyfish. This undulating movement was confirmed in a subsequent patient who underwent direct cystomyelography of their SAC (Video 2).

VIDEO 2. Clip showing an AP view highlighting the characteristic jellyfish sign, representing the containment of contrast and undulating flow pattern present within the cyst. Copyright Ram V. S. R.

Chavali. Published with permission. Click here to view.

A prior report of an intracranial temporal lobe arachnoid cyst described the margin of cortex that interfaced with the arachnoid cyst as undulating, but this was based on static MRI inference and described the contours of the cortex rather than the dynamic rhythmic nature of the cyst wall. ${ }^{12}$ A leading mechanism postulated to result in SAC expansion is via a unidirectional valve. ${ }^{2}$ We believe the pulsatile movement demonstrates the previously proposed waterhammer effect of the "trapped" CSF striking the end of the cyst wall, which, in fact, represents a windsock deformity of the thecal sac. The visualization of contrast freely flowing into the cyst without any backflow reflux through the cyst into the subarachnoid space is further evidence of a 1-way ball-valve mechanism.
We also report, for the first time, a percutaneous treatment option via balloon fenestration of an SAC. In the prior report by Takahashi et al., fenestration occurred via a simple puncture of the cyst wall through its anterior aspect, and then along a straight trajectory, the investigators pushed the needle through the other side until coming in contact with the osseous surface of the vertebral body. ${ }^{14}$ This technique was successful in a straightforward small and simple cyst; however, in larger cysts that have more robust communication with the subarachnoid space, a simple aspiration may not work as the cyst wall may collapse over the small puncture sites and lead to recurrent cyst formation as had initially occurred in this patient. The technique of using a thin flexible wire to pierce the cyst followed by balloon inflation parallels that used in endoscopic third ventriculostomy (ETV), in which a semi-blunt dissector is passed through the floor of the third ventricle followed by dilation with a 2- or 3-Fr balloon catheter. ${ }^{5}$ It is important to deflate the balloon prior to returning the catheter into the cyst as otherwise it may tear the wall (or the floor of the third ventricle in the case of ETV) and result in bleeding. While in ETV bleeding can be monitored by direct visualization through the endoscope, during percutaneous fenestration, monitoring is performed via observation of clear CSF return from the accessory port of the balloon catheter. Additional measures to improve safety during this procedure include "awake" anesthesia in patients who will tolerate it or neuromonitoring in patients undergoing general anesthesia. As occurred in our patient, recurrence of the cyst is possible. Similar to ETV failure, the likely mechanism is related to scar formation over the fenestration. One possible solution is repeat fenestration if the patient and surgeon decide to avoid open surgery. We anticipate that with improvements in visualization, such as small endoscopes introduced through the catheter sheaths, it may be possible to safely fenestrate the cysts in multiple locations and/or create a larger-diameter "window."

MRI has become the imaging modality of choice for diagnosing spinal cysts and CT myelography can confirm a communication between the cyst and the subarachnoid space. ${ }^{8}$ However, intradural SACs remain difficult to identify because compression and/or focal displacement may be the only imaging findings observed using these modalities. ${ }^{6}$ On CT myelography, depending on the degree of subarachnoid communication, contrast will typically fill the cyst to some extent, and in some cases may take up to several hours to fill, expending additional hospital resources. ${ }^{11}$ In addition, repeat delayed imaging adds potentially unnecessary radiation. In cases in which the cyst fills completely soon after contrast injection, it may be challenging to identify the cyst boundaries. Therefore, CT myelograms can have a high false-negative rate depending on the technique of intrathecal dye administration and image acquisition. Direct percutaneous cystography immediately and accurately delineates the margins of the cyst for surgical planning. Rather than relying on indirect evidence of cord displacement to outline the location of the cyst, percutaneous cystography allows one to directly observe the boundaries of the cyst. This can assist in surgical planning by allowing one to better target the laminectomy over the cyst itself rather than the segment 
of compressed spinal cord that may not correlate with the location of the cyst boundaries. The procedure could be performed immediately prior to surgery and conceivably the balloon or wire could be left in place as a radiopaque marker to delineate the inner cyst wall boundary, similar to needle localization for breast biopsy.

Limitations of this approach include the use of ionizing radiation. However, in contrast to other imaging modalities such as MRI, special MR-compatible equipment is not required, and this technique allows for true real-time imaging as there is no lag in image acquisition as there is in MRI. Another limitation includes the inability to visualize the operative anatomical site as is possible with endoscopy in ETV or the soft tissues with MRI in MRguided treatments. Repeatedly attempting to puncture the cyst puts the spinal cord and/or cauda equina at increased risk and therefore this procedure heavily relies on an experienced neurointerventional operator. Furthermore, this technique at present is limited to cysts with a dorsal component that is easily accessed, as intrathecal navigation anteriorly is challenging and risky to the spinal cord and/ or cauda equina. Lastly, as occurred in our patient at 1 year, recurrence can occur in both an early and late time period, and therefore regular follow-up evaluation needs to be instituted following this procedure. Complications such as bleeding may be heralded by blood-tinged fluid from the side port of the balloon catheter.

\section{Conclusions}

We describe a simple technique to rapidly visualize and confirm the diagnosis of SAC and aid accurate presurgical localization. We believe the jellyfish sign is a new sine qua non of intradural SAC. Initial aspiration of CSF with some relief of symptoms may serve as a simple and new functional test, as was observed in our case. A possible percutaneous method to fenestrate an intradural SAC with readily available materials is presented. While we used 3D rotational fluoroscopy to help with needle trajectory planning, a standard biplane fluoroscopic approach with careful planning can also be used to perform the procedure. Not only does direct puncture of the cyst provide a potential means to treat SACs, but also we feel this approach represents an improvement over current standard myelography technique in patients with a suspected diagnosis of SAC based on MRI. Contrast applied directly at this site should also aid in simultaneously differentiating/identifying associated cord herniation.

\section{References}

1. Bartanusz V, Savage JG, Belayev A: Spinal cord herniation or intradural arachnoid cyst? Spine J 14:385, 2014

2. Basaldella L, Orvieto E, Dei Tos AP, Della Barbera M, Valente M, Longatti P: Causes of arachnoid cyst development and expansion. Neurosurg Focus 22(2):E4, 2007

3. Bassiouni H, Hunold A, Asgari S, Hübschen U, König HJ, Stolke D: Spinal intradural juxtamedullary cysts in the adult: surgical management and outcome. Neurosurgery 55:13521360, 2004

4. Bellavia R, King JT Jr, Naheedy MH, Lewin JS: Percutaneous aspiration of an intradural/extradural thoracic arachnoid cyst: use of MR imaging guidance. J Vasc Interv Radiol 11:369-372, 2000

5. Brockmeyer D: Techniques of endoscopic third ventriculostomy. Neurosurg Clin N Am 15:51-59, 2004

6. Haber MD, Nguyen DD, Li S: Differentiation of idiopathic spinal cord herniation from CSF-isointense intraspinal extramedullary lesions displacing the cord. Radiographics 34:313-329, 2014

7. Jain SK, Sundar IV, Sharma V, Goel RS: Lumbosacral arachnoid cyst with tethered cord: a rare case report. J Craniovertebr Junction Spine 3:73-75, 2012

8. Khosla A, Wippold FJ II: CT myelography and MR imaging of extramedullary cysts of the spinal canal in adult and pediatric patients. AJR Am J Roentgenol 178:201-207, 2002

9. Kumar K, Malik S, Schulte PA: Symptomatic spinal arachnoid cysts: report of two cases with review of the literature. Spine (Phila Pa 1976) 28:E25-E29, 2003

10. Liu JK, Cole CD, Kan P, Schmidt MH: Spinal extradural arachnoid cysts: clinical, radiological, and surgical features. Neurosurg Focus 22(2):E6, 2007

11. Osenbach RK, Godersky JC, Traynelis VC, Schelper RD: Intradural extramedullary cysts of the spinal canal: clinical presentation, radiographic diagnosis, and surgical management. Neurosurgery 30:35-42, 1992

12. Robertson SJ, Wolpert SM, Runge VM: MR imaging of middle cranial fossa arachnoid cysts: temporal lobe agenesis syndrome revisited. AJNR Am J Neuroradiol 10:10071010, 1989

13. Silbergleit R, Brunberg JA, Patel SC, Mehta BA, Aravapalli SR: Imaging of spinal intradural arachnoid cysts: MRI, myelography and CT. Neuroradiology 40:664-668, 1998

14. Takahashi S, Morikawa S, Egawa M, Saruhashi Y, Matsusue Y: Magnetic resonance imaging-guided percutaneous fenestration of a cervical intradural cyst. Case report. J Neurosurg 99 (3 Suppl):313-315, 2003

15. Wang MY, Levi AD, Green BA: Intradural spinal arachnoid cysts in adults. Surg Neurol 60:49-56, 2003

\section{Disclosures}

Dr. Chi reports being a consultant to K2M and DePuy Synthes.

\section{Author Contributions}

Conception and design: Chavali. Acquisition of data: all authors. Analysis and interpretation of data: all authors. Drafting the article: all authors. Critically revising the article: all authors. Reviewed submitted version of manuscript: all authors.

\section{Supplemental Information \\ Videos}

Video 1. https://vimeo.com/269643326.

Video 2. https://vimeo.com/269643351.

\section{Correspondence}

Ram V. S. R. Chavali: Brigham and Women's Hospital, Boston, MA.rchavali@partners.org. 